\title{
Insights into the mechanism of antiproliferative effects of primaquine-cinnamic acid conjugates on MCF-7 cells
}

\author{
PEACE MABETA ${ }^{1, *}$ \\ KRISTINA PAVIĆ ${ }^{2}$ \\ BRANKA ZORC ${ }^{2}$ \\ ${ }^{1}$ University of Pretoria \\ Faculty of Health Sciences \\ Department of Physiology \\ Pretoria 0007, South Africa \\ 2 University of Zagreb \\ Faculty of Pharmacy \\ and Biochemistry \\ HR-10 000 Zagreb, Croatia
}

Accepted January 292018

Published online February 1, 2018

\begin{abstract}
In our previous paper, we showed that three primaquine-cinnamic acid conjugates composed of primaquine (PQ) residue and cinnamic acid derivatives (CADs) bound directly by an amide linkage (1) or through an acylsemicarbazide spacer (2 and 3) had significant growth inhibitory effects on some cancer cell lines. Compound 1 induced significant growth inhibition in the colorectal adenocarcinoma (SW620), human breast adenocarcinoma (MCF-7) and cervical carcinoma (HeLa) cell lines, while compounds $\mathbf{2}$ and $\mathbf{3}$ selectively inhibited the growth of MCF-7 cells. To better understand the underlying mechanisms of action of these PQ-CADs, morphological studies of the effects of test compounds on MCF-7 cells were undertaken using haematoxylin and eosin stain. Further analysis to determine the effects of test compounds on caspase activity and on the levels of apoptosis proteins were undertaken using the enzyme-linked immunosorbent assay (ELISA). Haematoxylin and eosin staining revealed that compounds $\mathbf{1}$ and $\mathbf{3}$ induced morphological changes in MCF-7 cells characteristic of apoptosis, while 2-treated cells were in interphase. Cell cycle analysis showed that cells treated with 1 and $\mathbf{3}$ were in sub-G1, while cells treated with 2 were mainly in interphase (G1 phase). Further, the study showed that the treatment of MCF-7 cells with $\mathbf{1}$ and $\mathbf{3}$ resulted in poly ADP ribose polymerase (PARP) cleavage as well as caspase-9 activation, indicating that they induced apoptotic cell death. We further investigated their effects on two important processes during metastasis, namely, migration and invasion. Compounds $\mathbf{1}$ and 3 inhibited the migration and invasion of MCF-7 cells, while compound 2 had a marginal effect.
\end{abstract}

Keywords: primaquine, cinnamic acid, MCF-7, Bad, p53, PARP, migration and invasion

Cinnamic acid (CA) and cinnamic acid derivatives (CADs) are naturally occurring aromatic substances found in plants. Cinnamoyl moieties are present in a variety of metabolites of phenylpropanoid biosynthetic origin. They are important intermediates in the biosynthetic pathways of most of the aromatic natural products, including lignin, the second most

\footnotetext{
*Correspondence; e-mail: peace.mabeta@up.ac.za
} 
abundant biopolymer after cellulose (1). These ubiquitous plant constituents are also major sources of potential lead compounds in drug development. Various pharmacological activities of CADs have been reported: antioxidative, antimicrobial, antifungal, antiviral, antiinflammatory, hepatoprotective and hypolipidemic (2-5). CA scaffolds are present in several registered drugs: cinanserin and tranilast (antiallergic drugs) and ozagrel (antiplatelet agent) (Fig. 1) (6). $\alpha$-Cyano and $\alpha$-fluoro CADs were found to inhibit the growth of intraerythrocytic Plasmodium falciparum in culture (7), while $N$-cinnamoyl derivatives of aminoacridines and aminoquinolines are reported as dual-action antimalarials $(8,9)$ and $N$-cinnamoyl chloroquine analogs both as antimalarial and antiproliferative agents (10).

Anticancer potential of CADs has been also known for more than a century. The first report of cancer patients treated with CA and its hydroxyl derivative ortho-coumaric acid dates back to 1905. Since then, anticancer potential of CADs has been extensively studied and reported in a number of scientific papers (11-18). Two articles prepared by Su (19) and De (20) give a detailed overview of anticancer research of CADs. The efforts resulted in the discovery of two anticancer drugs, belinostat and panobinostat (Fig. 1), hydroxamic acids derived from CAD, efficient against haematological malignancies and solid tumours (21). They were recently approved for the treatment of peripheral T-cell lymphoma and multiple myeloma. Panobinostat and belinostat are non-selective histone deacetylase inhibitors (pan-HDAC inhibitors), which have been shown to impede multiple pathways implicated in cancer and reverse epigenetic events associated with cancer, thereby reducing survival and inducing apoptosis in cancer cells (22).

In our previous paper, we prepared and biologically evaluated novel primaquinecinnamic acid derivatives (PQ-CAD) of amide and acylsemicarbazide type and reported their strong antiproliferative activity against a panel of cancer cell lines or selectivity towards the adenocarcinoma cell line MCF-7 (23). PQ-CADs of amide type also exerted antimycobacterial and antimalarial activity (24), while acylsemicarbazide PQ-CADs were better antioxidative agents and showed significant inhibition of lipid peroxidation and soybean lipoxygenase. Three PQ-CADs attracted our special attention: (E)-3-(2fluorophenyl)- $N$-(4-(6-methoxyquinolin-8-ylamino) pentyl)acrylamide (1), (E)-1-(3-(4-methoxyphenyl)acryloyl)-4-(4-(6-methoxyquinolin-8-ylamino) pentyl)semicarbazide (2) and (E)-4-(4-(6-methoxyquinolin-8-ylamino)pentyl)-1-(3-(4-(trifluoro-methyl)phenyl)acryloyl) semicarbazide (3). The $o$-fluoro derivative 1 showed high activity against colorectal adenocarcinoma (SW620, IC $\left.C_{50}=0.3 \pm 0.1 \mu \mathrm{M}\right)$, human breast adenocarcinoma $\left(\mathrm{MCF}-7, I_{50}=1.1 \pm\right.$ $0.6 \mu \mathrm{M})$ and HeLa $\left(I C_{50}=2.1 \pm 2.1 \mu \mathrm{M}\right)$ cell lines, while acylsemicarbazide 2 , and especially

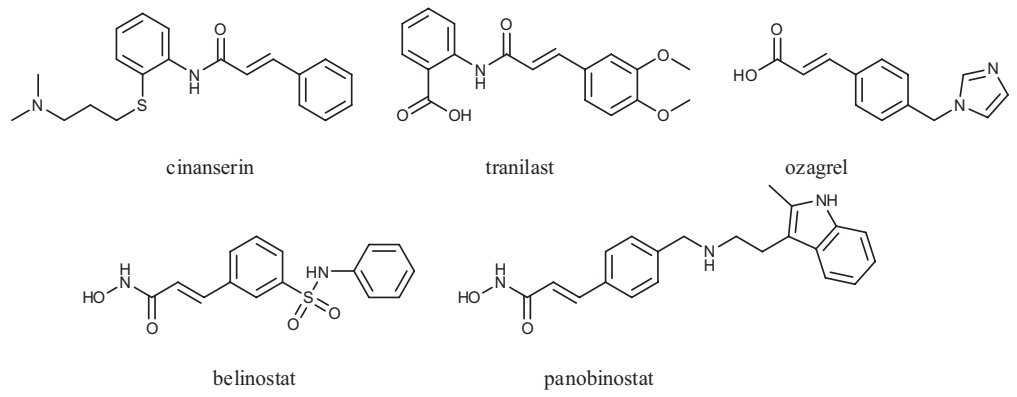

Fig. 1. Chemical structures of the approved drugs with the cinnamic acid scaffold. 
3 with methoxy- or trifluoromethyl- substituents in the para position showed high selectivity and high inhibitory activity against the MCF-7 cell line at micromolar (2) and nanomolar scales (3) $\left(I C_{50}=0.4 \pm 0.2\right.$ and $0.03 \pm 0.02 \mu \mathrm{M}$, respectively). In order to better elucidate the specific mechanism of the anti-cancer effects of PQ-CADs, further investigations of cell morphology, apoptosis proteins as well as cell migration and invasion were undertaken.

\section{EXPERIMENTAL}

\section{Chemistry}

\section{Reagents and equipment}

Melting points were measured on a Stuart Melting Point (SMP3) apparatus (Barloworld Scientific, UK) in open capillaries, with uncorrected values. IR spectra were recorded on an FTIR Perkin Elmer Paragon 500. ${ }^{1} \mathrm{H}$ and ${ }^{13} \mathrm{C}$ NMR spectra were recorded on NMR Avance 600 (Bruker, Germany) and Varian Inova 400 spectrometers at 300, 400 and $600 \mathrm{MHz}$ for ${ }^{1} \mathrm{H}$ and 75, 100 and $150 \mathrm{MHz}$ for ${ }^{13} \mathrm{C}$ nuclei, respectively. All compounds were routinely checked by TLC with Merck silica gel 60F-254 glass plates using the following solvent systems: petroleum ether/ethyl acetate/methanol 30:10:5, dichloromethane/methanol 97:3 and 95:5. Spots were visualized with short-wave UV light and iodine vapour. Column chromatography was performed on silica gel 0.063-0.200 mm (Kemika, Croatia) and $0.040-0.063 \mathrm{~mm}$ (Merck, Germany), with the same eluents as used in TLC.

All chemicals, solvents and biochemical reagents were of analytical grade and were purchased from Sigma-Aldrich (USA).

\section{Synthesis of compounds 1-3}

Compounds 1-3 were prepared following our procedure (23). Their analytical and spectral data were in full agreement with the previously published data.

\section{Biological evaluation}

\section{Cell culture}

The human breast adenocarcinoma cell line, MCF-7, purchased from the American Type Culture Collection (ATCC, USA) was grown in Dulbecco's Minimum Essential Medium (DMEM) (Sigma-Aldrich, Germany) supplemented with $10 \%$ heat-inactivated foetal bovine serum (FBS) (Invitrogen, USA) and $1 \%$ penicillin-streptomycin (Invitrogen, USA). The cell line was maintained in a $37^{\circ} \mathrm{C}$ incubator, under a humidified atmosphere containing $5 \% \mathrm{CO}_{2}$.

\section{Morphology studies}

MCF-7 cells were seeded on heat-sterilized cover slips in 6-well plates at a density of $3 \times 10^{5}$. After 24 hours, the cells were treated with compounds $1(1.1 \mu \mathrm{M}), 2(0.4 \mu \mathrm{M})$ and 3 $(0.03 \mu \mathrm{M})$ over a period of 24 hours. Control cells were exposed to $0.05 \%$ dimethyl sulfoxide (DMSO). Cells were stained with haematoxylin and eosin (H\&E) using a previously described method (28). The slides were viewed with an Olympus BX63 Light microscope 
and images were captured using an Olympus DP72 digital camera (Wirsam Scientific, SA). The mitotic index was obtained from the ratio of the number of cells in mitosis to the total number of cells.

\section{Apoptosis assay: Caspase-9 activity}

MCF-7 cells were seeded at a density of $3 \times 10^{5}$ cells/well and allowed to attach overnight. The cells were treated with compounds 1-3 at concentrations given above (1.1, 0.4 and $0.03 \mu \mathrm{M}$ ) for 24 hours. The compounds were diluted in medium to a final volume of 3 mL per well. Caspase-9 activity was measured using a commercially available kit according to the manufacturer's instructions (BioVision, USA).

\section{Apoptosis assay: Apoptosis protein levels}

Apoptosis proteins were measured using a PathScan Apoptosis Multi-Target Sandwich ELISA Kit (Cell Signaling Technology, USA) according to the manufacturer's instructions. Cells were seeded in 96-well plates at a density of $5 \times 10^{3}$ cells/well and treated with compounds $1(1.1 \mu \mathrm{M}), 2(0.4 \mu \mathrm{M})$ and $3(0.03 \mu \mathrm{M})$ or $0.05 \%$ (DMSO) for 24 hours. The cells were lysed with lysis buffer, and protein concentrations were measured to confirm equal loading onto an ELISA plate. Absorbance was read at $450 \mathrm{~nm}$ using a BioTek ELx800 Plate Reader (BioTek ${ }^{\circledR}$ Instruments, USA).

\section{Cell migration and invasion}

Cell migration and invasion experiments were performed as previously described (29). Briefly, using 16-well cell invasion and migration (CIM) plates (Roche Applied Science, SA), $160 \mu \mathrm{L}$ serum-free medium with or without a chemoattractant $(10 \mathrm{ng} / \mathrm{mL}$ fibroblast growth factor-2), was added to each well of the lower plate chamber. The plates were allowed to equilibrate at $37^{\circ} \mathrm{C}$ and a baseline measurement was taken. The cells were then added to the upper chamber of the CIM plate at a density of $6 \times 10^{3}$ cells/well and treated with compounds $1(1.1 \mu \mathrm{M}), 2(0.4 \mu \mathrm{M})$ and $3(0.03 \mu \mathrm{M})$ or $0.05 \%$ DMSO. The final volume of the solution in medium was $200 \mu \mathrm{L}$. Cell invasion and migration were monitored over 20 hours with an xCELLigence real time cell analysis (RTCA) DP instrument (Roche Applied Science, South Africa), and are represented as the cell index (CI). The analysis was performed using the RTCA software (vs. 1.2.1). Experiments were performed in triplicate.

\section{Statistical analysis}

Data were analyzed using One Way Analysis of Variance (ANOVA) and post-hoc Tukey's test. Results are expressed as mean values \pm SD of three separate experiments. A $p$-value of less than 0.05 was considered to be statistically significant.

\section{RESULTS AND DISCUSSION}

\section{Chemistry}

Three PQ-CADs were prepared and used for biological evaluation. In compound 1, PQ and $o$-fluorocinnamic acid are connected by an amide bond and in compounds 2 and 3 , PQ and $p$-methoxy or $p$-trifluoromethylcinnamic acid are bound by an acylsemicarba- 

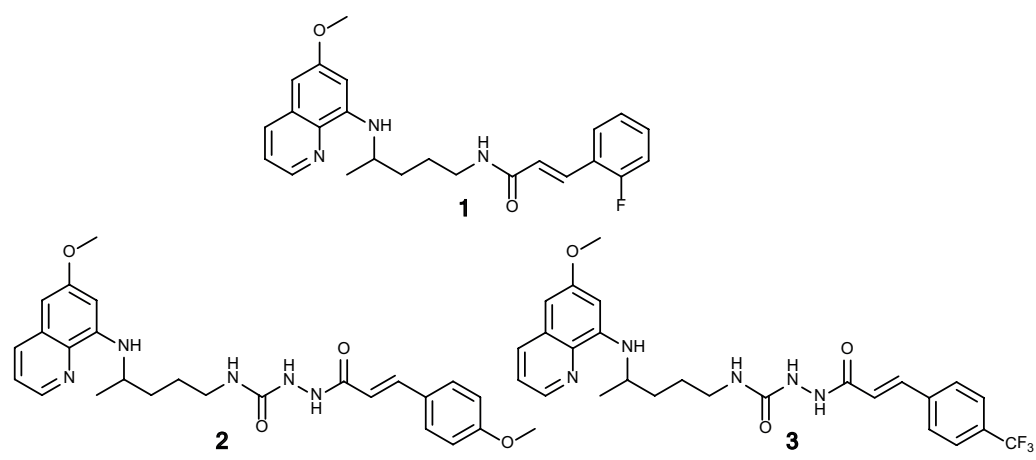

Fig. 2. Chemical structures of test compounds 1-3.

zide functional group built of PQ nitrogen atom, CONHNH spacer and carbonyl group originating from the corresponding cinnamic acid. Their structures are shown in Fig. 2.

Title compounds were prepared by synthetic procedures developed by us (23). Synthesis of cinnamamide $\mathbf{1}$ included a simple condensation reaction of PQ and $o$-fluorocinnamoyl chloride prepared from the corresponding cinnamic acid and thionyl chloride. Preparation of conjugates $\mathbf{2}$ and $\mathbf{3}$ was more complex and consisted of several reaction steps.

\section{Biological evaluation}

To better understand the mechanisms of action of the selected PQ-CAD derivatives on the MCF-7 cancer cell line, morphology, caspase activity and levels of apoptosis proteins p53, Bcl-2-associated death promoter (Bad) and poly ADP ribose polymerase (PARP), as well as cell migration and invasion, were studied following cell exposure to test compounds.

\section{Morphology}

Morphology of MCF-7 cells following exposure to test compounds revealed that cells were in mitosis, as evident from the representative images that show dividing cells in metaphase, characterized by coiled chromosomes aligned along the equator of the cell. Following exposure to 2, cells were predominantly in interphase as well as in metaphase and prophase, the early phases during mitosis associated with the condensation of chromatin threads, which are visible in the centre of the cell. Interphase occurs prior to cell division and morphological features during this phase include diffuse chromatin, observed in the images (Fig. 2). Cells treated with $\mathbf{1}$ and $\mathbf{3}$ were round in shape and showed hyper-condensed chromatin. Both of these morphological features are characteristic of apoptotic cell death. Flow cytometry was then employed for cell cycle analysis. During the analysis, endonuclear duplication, which occurs when DNA is replicated without apoptosis, was detected as cell debris. Cell cycle analysis also showed that $\mathbf{1}$ and $\mathbf{3}$ induced apoptosis in MCF-7 cells, observed from the high percentage of cells in sub-G1 (Fig. 4). Control cells, as well as cells treated with $\mathbf{2}$, were mainly in the first gap phase (G1) of the cell cycle. The G1 phase is a part of interphase during which the cell synthesizes mRNA and proteins. Cells treated with $\mathbf{1}$ appeared to have undergone a metaphase block, with subsequent induction of apoptosis (Fig. 4). 
a)

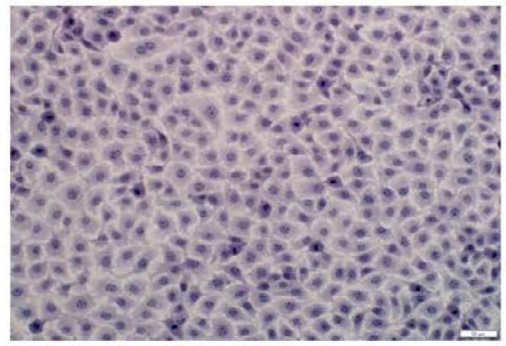

b)

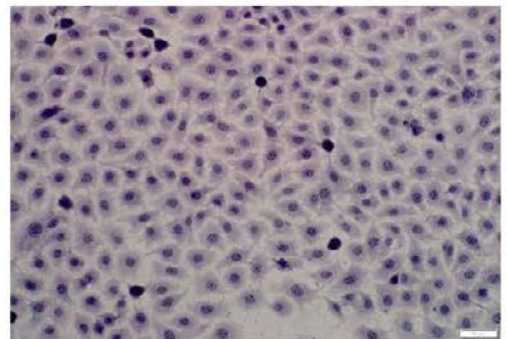

c)

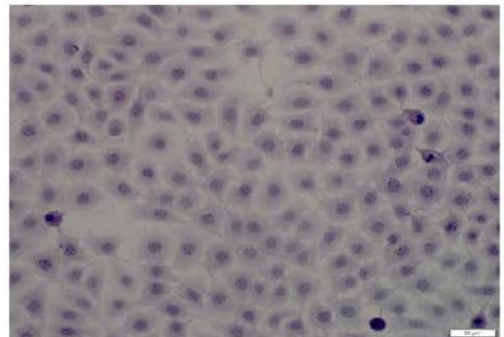

d)

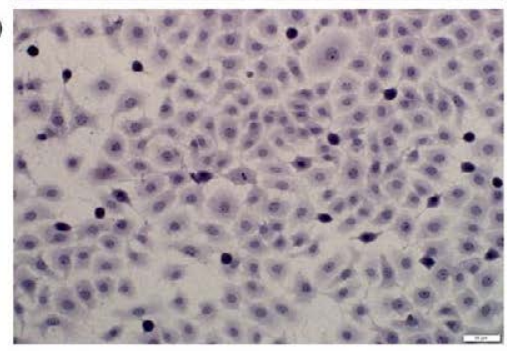

Bar $100 \mu \mathrm{m}$
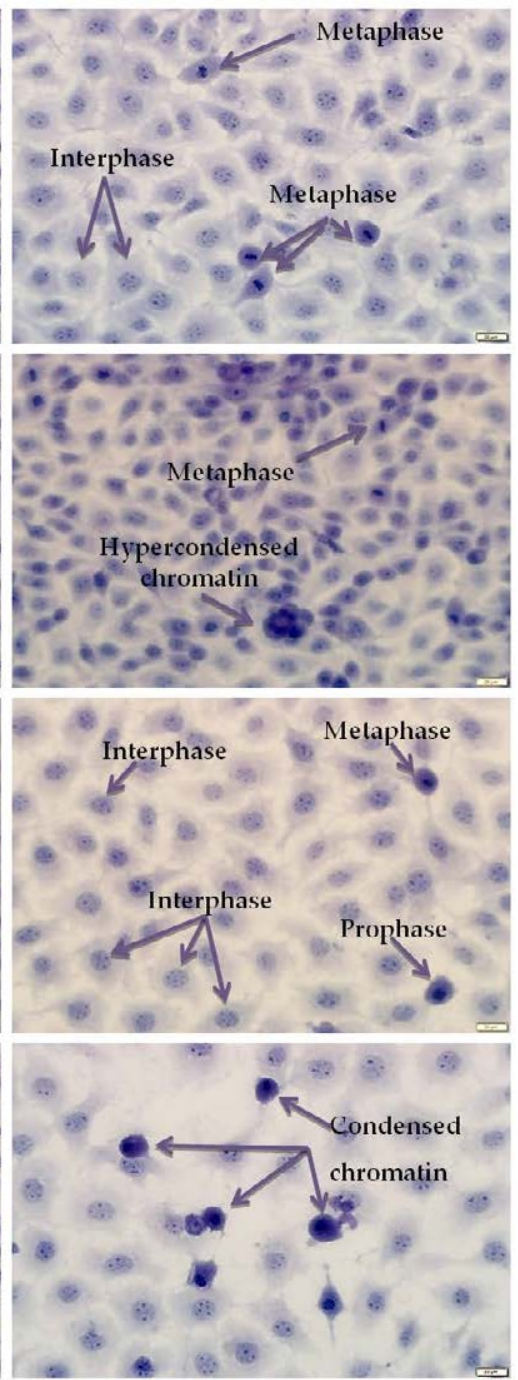

Bar $20 \mu \mathrm{m}$

Fig. 3. The effects of test compounds on the morphology of MCF-7 cells: a) control, b) compound 1, c) compound 2, d) compound 3 .

\section{Apoptosis}

To further investigate apoptotic induction, proteins that modulate apoptosis, namely, Bad, PARP and p53, were measured using a multi-target apoptosis detection kit. The kit also measures caspase- 3 activation: however, caspase- 3 was not detected in the samples and was thus not included in the results. Therefore, the activity of another protease, cas- 

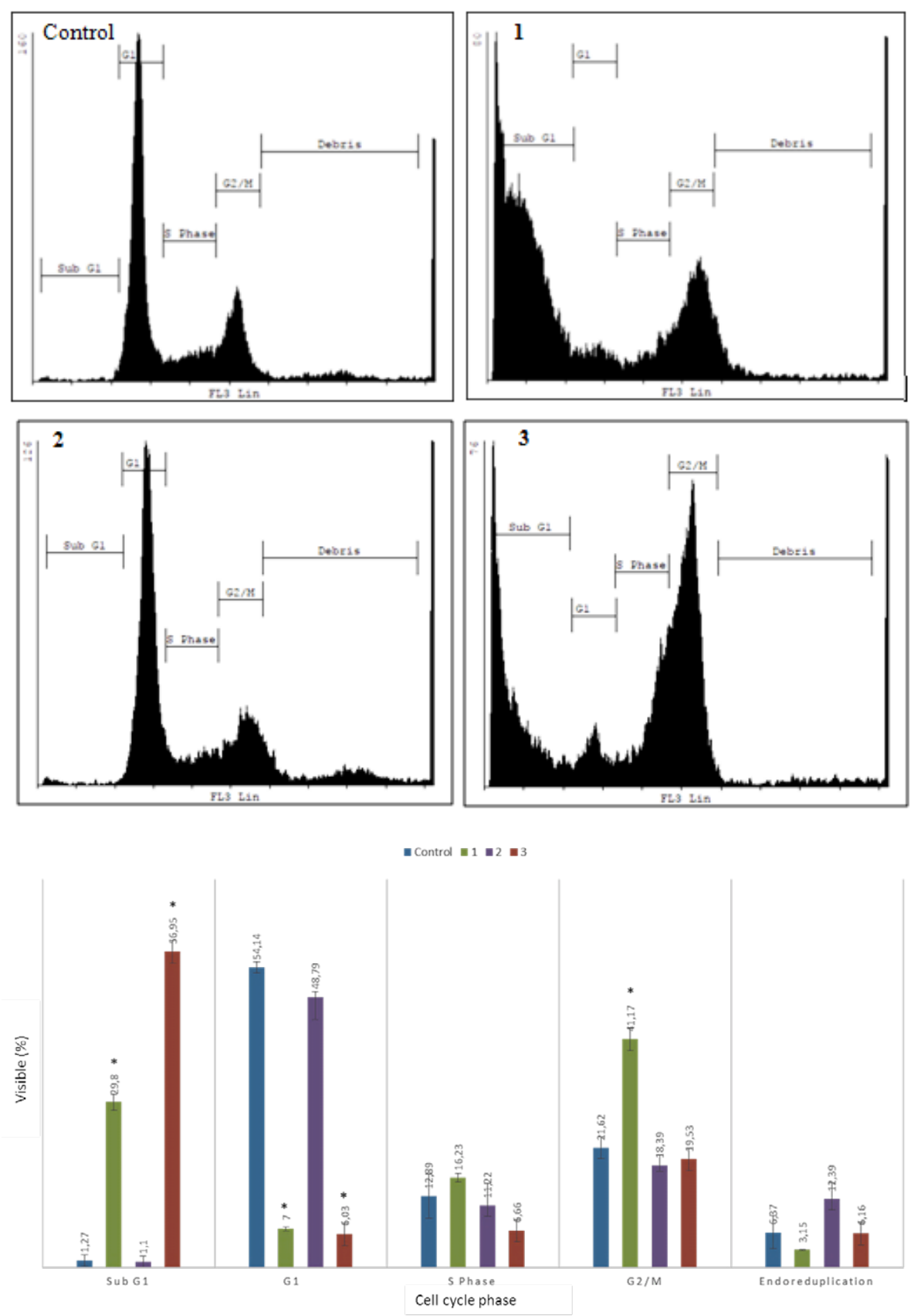

Fig. 4 . The effects of test compounds on the cell cycle. Results represent mean values \pm SD. Significant difference $v$ s. control, ${ }^{*} p<0.05$. 


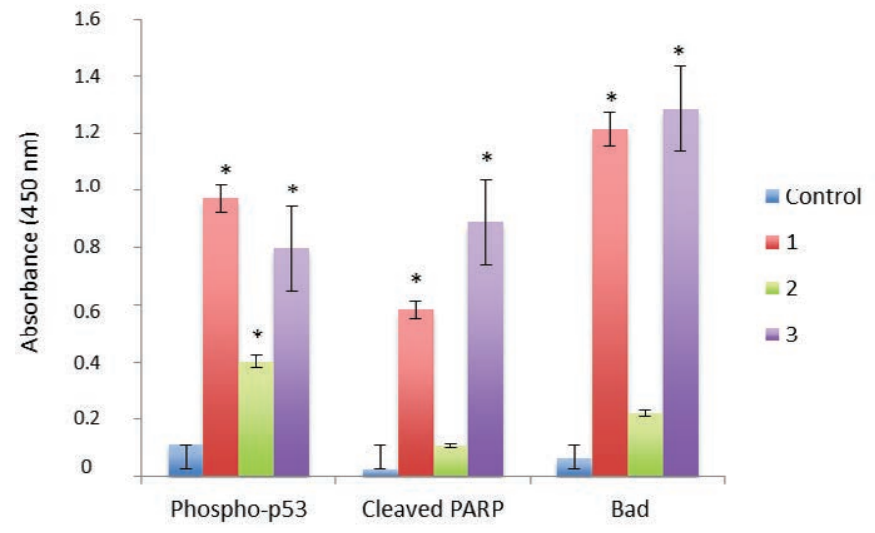

Fig. 5. The effects of test compounds on the levels of apoptosis signaling proteins. Results represent mean values \pm SEM. Significant difference $v$ s. control, ${ }^{*} p<0.05$.

pase-9, was measured. Results also showed an increase in p53 phosphorylation at Ser15 following the treatment of MCF-7 cells with test compounds (Fig. 5). p53 is activated through phosphorylation in response to cellular stress or insult and can induce cell growth arrest, activate DNA repair or induce apoptosis (25). Both $\mathbf{1}$ and $\mathbf{3}$ induced PARP-cleavage. PARP is a $116 \mathrm{kDa}$ protein involved in the repair of DNA $(25,26)$, while cleaved PARP is an executor of apoptosis (26). In addition, high levels of Bad were measured in $\mathbf{1}$ and $\mathbf{3}$ treated cells (Fig. 5). Bad is a member of the B-cell lymphoma 2 (Bcl2) family and it forms heterodimers with anti-apoptotic proteins B-cell lymphoma-extra large (Bcl-xL) and Bcl-2, thus

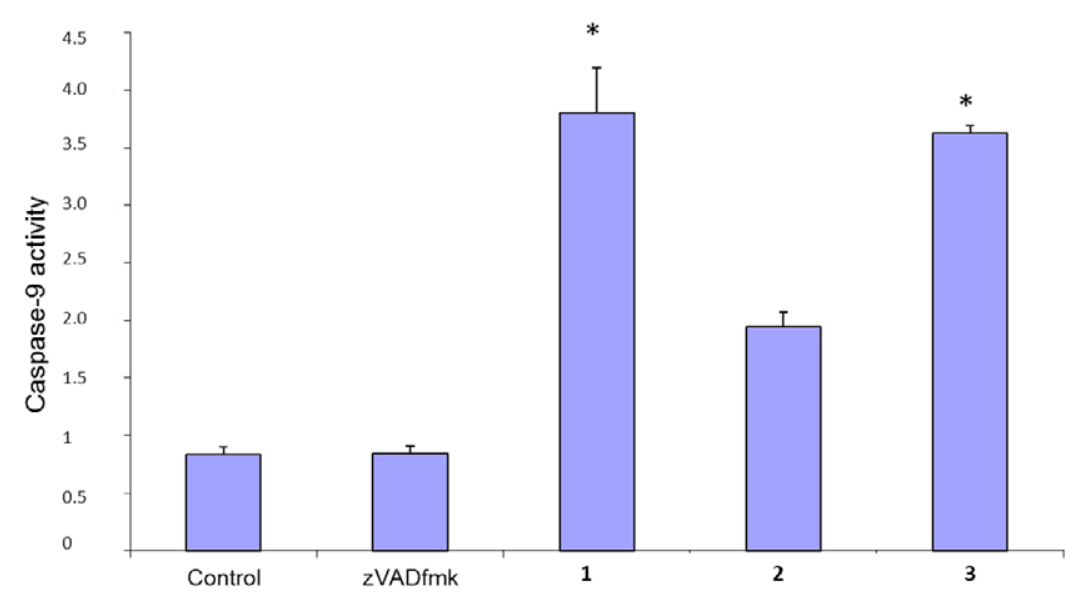

Fig. 6. Effects of test compounds on the activity of caspase-9. Results represent mean values \pm SD. Significant difference $v$ s. control, ${ }^{*} p<0.05$. 


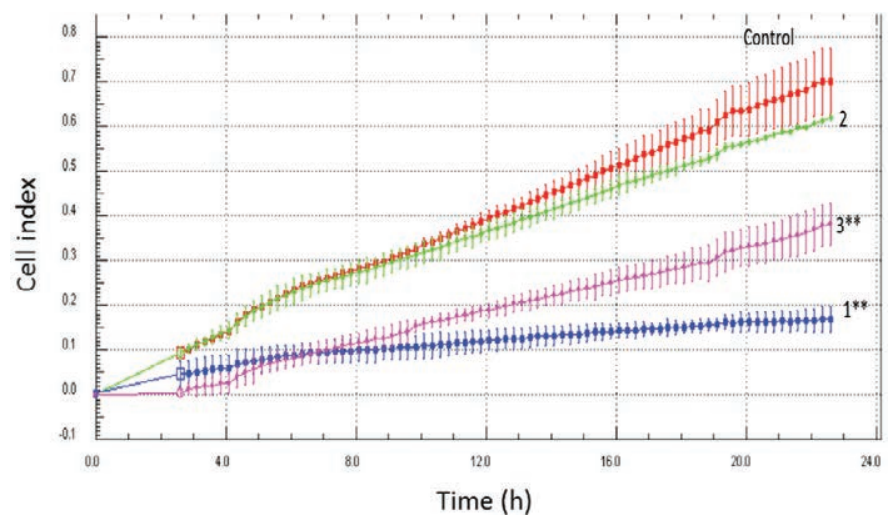

Fig. 7. The effects of test compounds on MCF-7 cell invasion and migration in response to fibroblast growth factor-2 stimulation. Values are expressed as the mean cell index \pm SD. Significant difference vs. control, ${ }^{* *} p<0.001$.

preventing their apoptosis suppressive role (27). Compounds $\mathbf{1}$ and $\mathbf{3}$ also stimulated caspase-9 activity (Fig. 6). Therefore, 1 and 3 induced apoptosis in MCF-7 cells partly by inducing increased Bad expression, caspase- 9 activation and PARP-cleavage.

\section{Cell invasion and migration}

The invasion and migration of MCF-7 cells in response to treatment with test compounds were monitored over 20 hours by measuring electrical impedance using the xCELLigence system. The results showed that 2 resulted in a marginal decrease in cell invasion and migration. On the other hand, 1 and 3 induced a pronounced inhibition of the migration of MCF-7 cells and their invasion into the lower chamber of the CIM plate (Fig. 7).

\section{CONCLUSIONS}

The studies carried out in the present work suggest that compounds $\mathbf{1}$ and $\mathbf{3}$ inhibit breast cancer cell growth by inducing apoptotic cell death in MCF-7 cells. On the other hand, compound 2 does not directly induce apoptosis, but inhibits cell growth, possibly by inducing cell cycle arrest in interphase. The difference in their biological response is a consequence of their chemical diversity. All three compounds share the same PQ and cinnamic acid moieties, but differ in spacers and substituents attached to the cinnamic acid benzene ring. Compounds 1 and 3 have a different spacer, but a similar halogen substituent, fluoro and trifluoromethyl, respectively. The fact that they share a similar biological response suggests that halogen substitution is more important than the spacer. Further experiments are needed to better understand the underlying mechanism of compound 2 . Compounds 1 and 3 inhibited MCF-7 migration and invasion as well. Both migration and invasion contribute to the metastatic spreading of cancer cells to other parts of the body. Therefore, these lead compounds require further testing in a mouse xenograft model of breast cancer to better determine their potential as anticancer agents. It is noteworthy that 
both compounds possess a fluorine atom (1) or organofluorine group (3) in their structures. Introduction of such moieties into biologically active compounds is often considered to induce beneficial pharmacological properties $(30,31)$. In addition, the number of atoms, relative molecular mass, $\log P, \mathrm{H}$-bond donor and acceptors, Lipinski score, molecular refractivity and polar surface area of all three tested compounds are in full agreement with the generally accepted rules for prospective small molecular drugs (23). If all the above facts are summarized, compounds $\mathbf{1}$ and $\mathbf{3}$ can be considered to be good candidates for further drug development.

Acknowledgment. - Support to this study by the Croatian Science Foundation (project number IP-2014-09-1501), as well as by the National Research Foundation and the University of Pretoria are gratefully acknowledged.

Acronyms. - Bad - Bcl-2-associated death promoter; Bcl-2 B-cell lymphoma 2; Bcl-xL - B-cell lymphoma-extra large; CA - cinnamic acid; CAD - cinnamic acid derivative; CI - cell index; CIM cell invasion/migration; DMM - Dulbecco's Minimum Essential Medium; DMSO - dimethyl sulfoxide; ELISA - enzyme-linked immunosorbent assay; HDAC - histone deacetylase; HeLa - cervical carcinoma cell line; H\&E - haematoxylin and eosin stain; MCF-7 - human breast adenocarcinoma cell line; PARP - poly ADP ribose polymerase; PQ - primaquine; RTCA - real time cell analysis; SW620 -colorectal adenocarcinoma.

\section{REFERENCES}

1. P. Sharma, Cinnamic acid derivatives: A new chapter of various pharmacological activities, J. Chem. Pharm. Res. 3 (2011) 403-423.

2. J. D. Guzman, Natural cinnamic acids, synthetic derivatives and hybrids with antimicrobial activity, Molecules 19 (2014) 292-349; https://doi.org/10.3390/molecules191219292

3. R. Lone, R. Shuab and K. K. Koul, Role of cinnamate and cinnamate derivatives in pharmacology, Glob. J. Pharmacol. 8 (2014) 328-335; https://doi.org/10.5829/idosi.gjp.2014.8.3.83132

4. M. Sova, Antioxidant and antimicrobial activities of cinnamic acid derivatives, Mini Rev. Med. Chem. 12 (2012) 749-767; https://doi.org/10.2174/138955712801264792

5. M. K. Lee, Y. B. Park, S. S. Moon, S. H. Bok, D. J. Kim, T. Y. Ha, T. S. Jeong, K. S. Jeong and M. S. Choi, Hypocholesterolemic and antioxidant properties of 3-(4-hydroxyl)propanoic acid derivatives in high-cholesterol fed rats, Chem.-Biol. Interact. 170 (2007) 9-19; https://doi.org/10.1016/j.cbi.2007.06.037

6. J. Zhang, J. Yang, X. Chang, C. Zhang, H. Zhou and M. Liu, Ozagrel for acute ischemic stroke: a meta-analysis of data from randomized controlled trials, Neurol. Res. 34 (2012) 346-353; https://doi. org/10.1179/1743132812Y.0000000022

7. J. Kanaani and H Ginsburg, Effects of cinnamic acid derivatives on in vitro growth of Plasmodium falciparum and on the permeability of the membrane of malaria-infected erythrocytes, Antimicrob. Agents Chemother. 36 (1992) 1102-1108.

8. B. Pérez, C. Teixeira, A. S. Gomes, I. S. Albuquerque, J. Gut, P. J. Rosenthal, M. Prudêncio and P. Gomes, In vitro efficiency of 9-(N-cinnamoylbutyl)aminoacridines against blood- and liver-stage malaria parasites, Bioorg. Med. Chem. Lett. 23 (2013) 610-613; https://doi.org/10.1016/j.bmcl.2012.12.032

9. B. C. Pérez, C. Teixeira, M. Figueiras, J. Gut, P. J. Rosenthal, J. R. B. Gomes and P. Gomes, Novel cinnamic acid/4-aminoquinoline conjugates bearing non-proteinogenic amino acids: Towards the development of potential dual action antimalarials, Eur. J. Med. Chem. 54 (2012) 887-899; https://doi. org/10.1016/j.ejmech.2012.05.022

10. B. C. Pérez, I. Fernandes, N. Mateus, C. Teixeira and P. Gomes, Recycling antimalarial leads for cancer: Antiproliferative properties of $\mathrm{N}$-cinnamoyl chloroquine analogues, Bioorg. Med. Chem. Lett. 23 (2013) 6769-6772; https://doi.org/10.1016/j.bmcl.2013.10.025 
11. K. Frenkel, H. Wei, R. Bhimani, J. Ye, J. A. Zadunaisky, M.-T. Huang, T. Ferraro, A. H. Conney and D. Grunberger, Inhibition of tumor promoter-mediated processes in mouse skin and bovine lens by caffeic acid phenethyl ester, Cancer Res. 53 (1993) 1255-1261.

12. L. Liu,W. R. Hudgins, S. Shack, M. Q. Yin and D. Samid, Cinnamic acid: a natural product with potential use in cancer intervention, Int. J. Cancer 62 (1995) 345-350.

13. S. Mishima, Y. Ono, Y. Araki, Y. Akao and Y. Nozawa, Two related cinnamic acid derivatives from Brazilian honey bee propolis, baccharin and drupanin, induce growth inhibition in allografted sarcoma S-180 in mice, Biol. Pharm. Bull. 28 (2005) 1025-1030; https://doi.org/10.1248/bpb.28.1025

14. Y. Qian, H.-J. Zhang, H. Zhang, C. Xu, J. Zhao and H.-L. Zhu, Synthesis, molecular modeling, and biological evaluation of cinnamic acid metronidazole ester derivatives as novel anticancer agents, Bioorg. Med. Chem. 18 (2010) 4991-4996; https://doi.org/10.1016/j.bmc.2010.06.003

15. J. Dai and J. M. Russell, Plant phenolics: Extraction, analysis and their antioxidant and anticancer properties, Molecules 15 (2010) 7313-7352; https://doi.org/10.3390/molecules15107313

16. X.-H. Yang, Q. Wen, T.-T. Zhao, J. Sun, X. Li, M. Xing, X. Lu and H.-L. Zhu, Synthesis, biological evaluation, and molecular docking studies of cinnamic acyl 1,3,4-thiadiazole amide derivatives as novel antitubulin agents, Bioorg. Med. Chem. 20 (2012) 1181-1187; https://doi.org/10.1016/j. bmc.2011.12.057

17. D. P. Bezerra, C. Pessoa, M. O. de Moraes, N. Saker-Neto, E. R. Silveira and L. v. Costa-Lotufo, Overview of the therapeutic potential of piplartine (piperlongumine), Eur. J. Pharm. Sci. 48 (2013) 453-463; https://doi.org/10.1016/j.ejps.2012.12.003

18. C.-C. Xu, T. Deng, M.-L. Fan, W.-B. Lv, J.-H. Liu and B.-Y. Yu, Synthesis and in vitro antitumor evaluation of dihydroartemisinin-cinnamic acid ester derivatives, Eur. J. Med. Chem. 107 (2016) 192-203; https://doi.org/10.1016/j.ejmech.2015.11.003

19. P. Su, Y. Shi, J. Wang, X. Shen and J. Zhang, Anticancer agents derived from natural cinnamic acids, Anticancer Agents Med Chem. 15 (2015) 980-987; https://doi.org/10.2174/1871520615666150130111120

20. P. De, M. Baltas and F. Bedos-Belval, Cinnamic acid derivatives as anticancer agents - a review, Curr. Med. Chem. 18 (2011) 1672-1703; https://doi.org/10.2174/092986711795471347

21. J. A. Plumb, P. W. Finn, R. J. Williams, M. J. Bandara, M. R. Romero, C. J. Watkins, N. B. La Thangue and R. Brown, Pharmacodynamic response and inhibition of growth of human tumor xenografts by the novel histone deacetylase inhibitor PXD101, Mol. Cancer Ther. 2 (2003) 721-728.

22. P. Revill, N. Mealy, N. Serradell, J. Bolos and E. Rosa, Panobinistat, Drugs Fut. 32 (2007) 315-322; https://doi.org/10.1358/dof.2007.032.04.1094476

23. K. Pavić, I. Perković, P. Gilja, F. Kozlina, K. Ester, M. Kralj, D. Schols, D. Hadjipavlou-Litina, E. Pontiki and B. Zorc, Design, synthesis and biological evaluation of novel primaquine-cinnamic acid conjugates of amide and acylsemicarbazide type, Molecules 21 (2016) 1629-1653; https://doi. org/10.3390/molecules21121629

24. K. Pavić, I. Perković, Š. Pospíšilová, M. Machado, D. Fonthinha, M. Prudêncio, J. Jampilek. A. Coffey, L. Endersen, H. Rimac and B. Zorc, Primaquine hybrids as promising antimycobacterial and antimalarial agents, Eur. J. Med. Chem. 143 (2018) 769-779; https://doi.org/10.1016/j.ejmech.2017.11.083

25. Z. Herceg and Z. Q. Wang, Functions of poly (ADP-ribose) polymerase (PARP) in DNA repair, genomic integrity and cell death, Mutat. Res. 477 (2001) 97-110; https://doi.org/10.1016/S00275107(01)00111-7

26. I. Perković, M. Antunović, I. Marijanović, K. Pavić, K. Ester, M. Kralj, J. Vlainić, I. Kosalec, D. Schols, D. Hadjipavlou-Litina, E. Pontiki and B. Zorc, Novel urea and bis-urea primaquine derivatives with hydroxyphenyl and halogenphenyl substituents: Synthesis and biological evaluation, Eur. J. Med. Chem. 124 (2016) 622-636; https://doi.org/10.1016/j.ejmech.2016.08.021

27. S. Hector and J. H. Prehn, Apoptosis signalling proteins as prognostic biomarkers in colorectal cancer: A review, BBA-Rev. Cancer 1795 (2009) 117-129; https://doi.org/10.1016/j.bbcan.2008.12.002

28. P. Mabeta and M. S. Pepper, Inhibition of hemangioma development in a syngeneic mouse model correlates with Bcl-2 suppression and the inhibition of Akt kinase activity, Angiogenesis 15 (2012) 131-139; https://doi.org/10.1007/s10456-011-9248-7 
29. P. Mabeta, PF573,228 inhibits vascular tumor cell growth, migration as well as angiogenesis, induces apoptosis and abrogates PRAS40 and S6RP phosphorylation, Acta Pharm. 66 (2016) 399-410; https://doi.org/10.1515/acph-2016-0031

30. I. Ojima, D. Awasthi, L. Wei and K. Haranahalli, Strategic incorporation of fluorine in the drug discovery of new-generation antitubercular agents targeting bacterial cell division protein FtsZ, J. Fluorine Chem. 196 (2017) 44-56; https://doi.org/10.1016/j.jfluchem.2016.07.020

31. H.-J. Böhm, D. Banner, S. Bendels, M. Kansy, B. Kuhn, K. Müller, U. Obst-Sander and M. Stahl, Fluorine in medicinal chemistry, ChemBioChem 5 (2004) 637-643; https://doi.org/10.1002/ cbic. 200301023 